\title{
Heavy-lepton production in the minimal supersymmetric standard model
}

\author{
J. E. Cieza Montalvo \\ Instituto de Física-Universidade de São Paulo, Caixa Postal 20516-CEP01498 São Paulo, Brazil \\ O. J. P. Éboli* and S. F. Novaes ${ }^{\dagger}$ \\ Department of Physics, University of Wisconsin \\ Madison, Wisconsin 53706 \\ (Received 3 December 1991)
}

\begin{abstract}
We study the production of a charged-heavy-lepton pair considering the minimal supersymmetric standard model. We show that the cross section for the process $p p \rightarrow g g \rightarrow \ell^{+} \ell^{-}$is enhanced for large values of the ratio between the two-Higgs-doublet vacuum expectation values, in comparison with the standard model result. The gluon fusion mechanism is the most important contribution to the lepton pair production for $M_{\ell}>50 \mathrm{GeV}$.
\end{abstract}

PACS number(s): $13.85 . \mathrm{Qk}, 12.15 . \mathrm{Cc}, 14.60 . \mathrm{Ji}$

\section{INTRODUCTION}

One of the greatest puzzles in particle physics is the spectrum of elementary fermions. There is no theoretical explanation for the existence of several generations and for the values of the masses. Recently, it was established at the CERN $e^{+} e^{-}$collider LEP that the number of light neutrinos is three [1]. However this result does not rule out the possibility that further generations exist, provided that they are sufficiently heavy.

In this paper, we shall consider the possible existence of a new generation of fermions and study the production of heavy-charged-lepton pairs in hadronic collisions. Traditionally, the production of dileptons proceeds via the Drell-Yan, process, i.e., the quark fusion mechanism $\left(q \bar{q} \rightarrow \ell^{+} \ell^{-}\right)$. Nevertheless, in high-energy hadronic collisions we can take advantage of the high gluon luminosity to produce leptons. In fact, within the scope of the standard model, it has been shown [2] that the DrellYan mechanism is dominant only for lepton masses below 100-200 GeV, whereas heavier leptons are predominantly produced by gluon fusion. In this work we shall study the production of heavy-lepton pairs through gluon fusion, assuming the minimal supersymmetric extension of the standard model [3]. We shall demonstrate that for large $\tan \beta$ the cross section is greatly enhanced, yielding a large number of events which can be extract from the background.

The minimal supersymmetric standard model (MSSM) requires at least two Higgs doublets in order to generate mass for the up and down quarks. Therefore, it possesses a rich spectrum of Higgs bosons [4], which contains two

* Permanent address: Instituto de Física, Universidade de São Paulo, Caixa Postal 20516, CEP 01498 São Paulo, Brazil

${ }^{\dagger}$ Permanent address: Instituto de Física Teórica, Universidade Estadual Paulista, Rua Pamplona 145, CEP 01405 São Paulo, Brazil neutral scalars $\left(H_{1}\right.$ and $\left.H_{2}\right)$, a neutral pseudoscalar $\left(H_{3}\right)$, and two charged ones $\left(H^{ \pm}\right)$.

In a generic two-doublet model, there are seven independent parameters in the Higgs potential, which can be chosen to be the $W$ mass, the masses of the four physical scalars, the mixing angle $\alpha$, and $\tan \beta=v_{2} / v_{1}$, the ratio of the vacuum expectation values of the two doublets. However, supersymmetry reduces this number to three, which we take to be the heavier neutral-Higgs-boson mass $\left(M_{H_{1}}\right), M_{W}$, and $\tan \beta$. The other four parameters can be obtained through the relations

$$
\begin{aligned}
M_{H_{2}} & =\frac{M_{Z}^{2}-M_{H_{1}}^{2}}{M_{Z}^{2} \cos ^{2}(2 \beta)-M_{H_{1}}^{2}} \cos ^{2}(2 \beta), \\
M_{H_{3}}^{2} & =M_{H_{1}}^{2}+M_{H_{2}}^{2}-M_{Z}^{2}, \\
M_{H^{ \pm}}^{2} & =M_{H_{3}}^{2}+M_{W}^{2}, \\
\tan 2 \alpha & =\tan 2 \beta\left[\frac{M_{H_{3}}^{2}+M_{Z}^{2}}{M_{H_{3}}^{2}-M_{Z}^{2}}\right] .
\end{aligned}
$$

The angles $\alpha$ and $\beta$ are such that $0 \leq \beta \leq \pi / 2$ and $-\pi / 2 \leq \alpha \leq 0$. There are some experimental limits on the Higgs-boson masses coming from $e^{+} e^{-}$data [5]: $M_{H_{2}} \geq 41 \mathrm{GeV}$, and $M_{H_{3}} \geq 20 \mathrm{GeV}$.

The fourth-generation heavy leptons are supposed to form a weak-isospin doublet with the same electroweak couplings as the first three generations of leptons. The search for such a charged heavy lepton in $e^{+} e^{-}$collisions near the $Z^{0}$ peak [6] was able to establish a lower limit on its mass of $42.8 \mathrm{GeV}$. On the other hand, we can obtain theoretical upper bounds on the masses and parameters of the minimal supersymmetric model using unitarity constraints [7,8]. In analogy with Ref. [9], we can use the saturation of these constraints for treeapproximation amplitudes to determine bounds on the fermion masses, beyond which the perturbation expansion breaks down. The analysis of the $J=0$ partial wave amplitude for the reaction $F_{i} \bar{F}_{i} \rightarrow F_{j} \bar{F}_{j}$, involving heavy fermions, constraints the mass of charged heavy lepton to satisfy 


$$
M_{\ell} \leq\left(\frac{4 \sqrt{2} \pi}{G_{F}}\right)^{1 / 2} \cos \beta=1200 \cos \beta \quad(\mathrm{GeV}) .
$$

Similarly, if we consider a new generation of heavy quarks $(U, D)$ their masses are also constrained by unitarity and must obey the relations

$$
\begin{aligned}
\left(\begin{array}{l}
M_{U} \\
M_{D}
\end{array}\right) & \leq\left(\frac{4 \sqrt{2} \pi}{3 G_{F}}\right)^{1 / 2}\left(\begin{array}{l}
\sin \beta \\
\cos \beta
\end{array}\right) \\
& =710\left(\begin{array}{c}
\sin \beta \\
\cos \beta
\end{array}\right) \mathrm{GeV} .
\end{aligned}
$$

For $\tan \beta=3(5)$, we should have $M_{\ell}<390(240) \mathrm{GeV}$, $M_{U}<670(700) \mathrm{GeV}$, and $M_{D}<225(140) \mathrm{GeV}$.

This paper is organized as follows. Section II contains the cross section for the subprocess $g g \rightarrow \ell^{+} \ell^{-}$, as well as the presentation of some interesting features of this process. Our results for the cross section $p p \rightarrow \ell^{+} \ell^{-}$are presented in Sec. III, where we also state our conclusions. In the Appendix, we give all the relevant coupling constants.

\section{SUBPROCESS CROSS SECTION}

Figure 1 shows the Feynman diagrams that contribute to the subprocess $g g \rightarrow \ell^{+} \ell^{-}$. The connection between the initial gluons and the weakly interacting leptons occurs, in the MSSM, via a loop of quarks and squarks, since these are the only particles with both strong and electroweak charges. Moreover, since the final state is neutral the $s$ channel involves only the exchange of the three neutral Higgs bosons $\left(H_{1}, H_{2}\right.$, and $\left.H_{3}\right)$ and the $Z^{0}$. The exchange of a photon is not allowed by $C$ conserva-

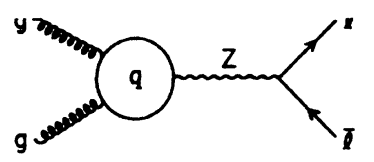

(a)



(b)



(c)



+ crossed diagrams

FIG. 1. Feynman diagrams contributing to the process $g g \rightarrow \ell^{+} \ell^{-}$.

tion (Furry's theorem), which also indicates that only the axial-vector couplings of the $Z^{0}$ contribute to this process.

In order to make explicit the different contributions to the elementary cross section, we will present them separately. The quark-loop contributions involve $Z^{0}$ [Fig. 1(a)], and the Higgs-boson [Fig. 1(b)] exchanges in the $s$ channel, yielding

$$
\begin{aligned}
\hat{\sigma}_{Z}^{q} & =\frac{\alpha^{2} \alpha_{s}^{2}}{512 \pi \sin ^{4} \theta_{W}} \frac{M_{\ell}^{2}}{M_{W}^{4}} \beta_{\ell}\left|\sum_{q=u, d} T_{3}^{q}\left(1+2 \lambda_{q} I_{q}\right)\right|^{2}, \\
\hat{\sigma}_{H_{1}+H_{2}}^{q} & =\frac{\alpha^{2} \alpha_{s}^{2}}{512 \pi \sin ^{4} \theta_{W}} \frac{M_{\ell}^{2}}{M_{W}^{4}} \beta_{\ell}^{3}\left|\sum_{i=1,2} \eta_{\ell}^{(i)} \chi^{(i)}(\hat{s}) \sum_{q=u, d} \eta_{q}^{(i)} m_{q}^{2}\left[2+\left(4 \lambda_{q}-1\right) I_{q}\right]\right|^{2}, \\
\hat{\sigma}_{H_{3}}^{q} & =\frac{\alpha^{2} \alpha_{s}^{2}}{512 \pi \sin ^{4} \theta_{W}} \frac{M_{\ell}^{2}}{M_{W}^{4}} \beta_{\ell}\left(\eta_{\ell}^{(3)}\right)^{2}\left|\chi^{(3)}(\hat{s})\right|^{2}\left|\sum_{q=u, d} \eta_{q}^{(3)} m_{q}^{2} I_{q}\right|^{2}, \\
\hat{\sigma}_{Z-H_{3}}^{q} & =-\frac{\alpha^{2} \alpha_{s}^{2}}{256 \pi \sin ^{4} \theta_{W}} \frac{M_{\ell}^{2}}{M_{W}^{4}} \beta_{\ell} \eta_{\ell}^{(3)} \operatorname{Re}\left[\chi^{(3)}(\hat{s}) \sum_{q=u, d} \eta_{q}^{(3)} m_{q}^{2} I_{q} \sum_{q^{\prime}=u, d} T_{3}^{q^{\prime}}\left(1+2 \lambda_{q^{\prime}} I_{q^{\prime}}^{*}\right)\right],
\end{aligned}
$$

where the summations run over all generations. $T_{3}^{q}$ is the quark weak isospin $\left[T_{3}^{u(d)}=+(-) 1 / 2\right], \beta_{\ell}=$ $\sqrt{1-4 M_{\ell}^{2} / \hat{s}}$ is the lepton velocity in the c.m. of the subprocess, and Re stands for the real part of the expression. The loop function $I_{i} \equiv I\left(\lambda_{i}=m_{i}^{2} / \hat{s}\right)$ and the couplings constants $\eta_{f}^{(i)}$, which are functions of the angles $\alpha$ and $\beta$, are given in the Appendix. We have also defined

$$
\chi^{(i)}(\hat{s})=\frac{1}{\hat{s}-M_{H_{i}}^{2}+i M_{H_{i}} \Gamma_{H_{i}}}
$$

with $\Gamma_{H_{i}}$ being the Higgs-boson total width.

The diagram with a $Z^{0}$ in the $s$ channel does not exhibit the resonance for $\hat{s}=M_{Z}^{2}$, since the on-shell production of a massive spin-one particle on shell from two massless spin-one particles is forbidden (Yang's theorem [10]). Furthermore, the effective coupling of the gluons 
to the $Z^{0}$ is proportional to $\partial_{\mu} Z^{\mu}$, where $Z^{\mu}$ is the vector boson field. This leads to an $Z^{0}$ amplitude which is proportional to $\partial_{\mu} J_{\ell}^{\mu}$, where $J_{\ell}^{\mu}$ is the neutral current associated with the lepton $\ell$. Therefore, this diagram is proportional to $M_{\ell}$, as can be seen from the above expressions.

The contributions coming from the Higgs boson are also proportional to the lepton mass, and exhibit the resonance peaks associated with the three neutral Higgs bosons. Because of the behavior of the loop function $I_{i}$, the contribution of the quark loop to the lepton pair production via the Higgs bosons has a peak centered at $\sqrt{\hat{s}}=2.5 m_{q}$ for $H_{1,2}$ and $\sqrt{\hat{s}}=2 m_{q}$ for $H_{3}$. The interference of the $Z^{0}$ and $H_{3}$ diagrams with the $H_{1}$ and $H_{2}$ ones vanishes, since the first two are antisymmetric in the gluon polarizations, while the latter are symmetric.

The squark-loop contribution [Figs. 1(c) and 1(d)] contains only the scalar-Higgs-boson exchange in the $s$ channel. Here again $C$ conservation forbids the coupling of the gluons to $\gamma$ and $Z^{0}$. There is not a $H_{3}$ contribution in this case, since the vertices $H_{3}-\tilde{q}_{L}-\tilde{q}_{L}$ and $H_{3}-\tilde{q}_{R}-\tilde{q}_{R}$ are absent. The scalar-quark loop contribution is given by

$$
\hat{\sigma}_{H_{1}+H_{2}}^{\tilde{q}}=\frac{\alpha^{2} \alpha_{s}^{2}}{512 \pi \sin ^{4} \theta_{W} \cos ^{4} \theta_{W}} M_{\ell}^{2} \beta_{\ell}^{3}\left|\sum_{i=1,2} \eta_{\ell}^{(i)} \chi^{(i)}(\hat{s}) \sum_{\tilde{q}=\tilde{u}, \tilde{d}} \sum_{k=L, R} \tilde{\eta}_{\tilde{q}_{k}}^{(i)}\left(1+2 \lambda_{\tilde{q}_{k}} I_{\tilde{q}_{k}}\right)\right|^{2}
$$

where the summations range over all scalar quarks and the couplings $\tilde{\eta}_{\tilde{q}}^{(i)}$ are given in the Appendix. The interference between the quark and squark loop contributions is given by

$$
\begin{aligned}
\hat{\sigma}_{H_{1}+H_{2}}^{q-\tilde{q}}=- & \frac{\alpha^{2} \alpha_{s}^{2}}{256 \pi \sin ^{4} \theta_{W} \cos ^{4} \theta_{W}}\left(\frac{M_{\ell}}{M_{Z}}\right)^{2} \\
& \times \beta_{\ell}^{3} \operatorname{Re}\left\{\sum_{i=1,2} \eta_{\ell}^{(i)} \chi^{(i)}(\hat{s})\right. \\
& \left.\quad \times \sum_{q=u, d} \eta_{q}^{(i)} m_{q}^{2}\left[2+\left(4 \lambda_{q}-1\right) I_{q}\right] \sum_{j=1,2} \eta_{\ell}^{(j)} \chi^{*(j)}(\hat{s}) \sum_{\tilde{q}=\tilde{u}, \tilde{d}} \sum_{k=L, R} \tilde{\eta}_{\tilde{q}_{k}}^{(j)}\left(1+2 \lambda_{\tilde{q}_{k}} I_{\tilde{q}_{k}}^{*}\right)\right\} .
\end{aligned}
$$

Since the expressions for the subprocess cross section are rather long and complicated, let us analyze it in some limits, in order to gain some insight about its behavior. When we assume only light particles running in the loop, the contribution to the cross section that is proportional to $\lambda_{i} I_{i}$ goes to zero. The contribution $\hat{\sigma}_{H_{1}+H_{2}}^{\tilde{q}}$ also goes to zero since, for $m_{q} / M_{Z} \simeq 0$, we have $\sum_{i=1,2} \sum_{\tilde{q}=\tilde{u}, \tilde{d}} \sum_{k=L, R} \tilde{\eta}_{\tilde{q}_{k}}^{(i)}=0$ (see the Appendix).

Taking into account a heavy generation, we must consider the large- $\lambda_{i}$ limit. In this case, we learn that the contribution involving the $Z^{0}$ are proportional to the mass split of the quark doublet due to the presence of $T_{3}^{q}$,

$$
\begin{aligned}
\hat{\sigma}_{Z} \equiv \hat{\sigma}_{Z}^{q}+\hat{\sigma}_{Z-H_{3}}^{q} \rightarrow & \frac{\alpha^{2} \alpha_{s}^{2}}{12288 \pi \sin ^{4} \theta_{W}} \frac{M_{\ell}^{2}}{M_{W}^{4}} \\
& \times \beta_{\ell} \hat{s}\left(\frac{M_{U}^{2}-M_{D}^{2}}{M_{U}^{2} M_{D}^{2}}\right) \\
& \times\left[\frac{\hat{s}}{24}\left(\frac{M_{U}^{2}-M_{D}^{2}}{M_{U}^{2} M_{D}^{2}}\right)-\frac{1}{\cos ^{2} \beta}\right]
\end{aligned}
$$

and, neglecting the resonance effect of the Higgs bosons, we have

$$
\begin{aligned}
\hat{\sigma}^{q} \equiv \hat{\sigma}_{H_{1}+H_{2}}^{q}+\hat{\sigma}_{H_{3}}^{q} \rightarrow & \frac{\alpha^{2} \alpha_{s}^{2}}{512 \pi \sin ^{4} \theta_{W}} \frac{M_{\ell}^{2}}{M_{W}^{4}} \\
& \times \beta_{\ell} \frac{1}{\cos ^{4} \beta}\left(\frac{\beta_{\ell}^{2}}{9}+\frac{1}{4}\right) .
\end{aligned}
$$

Finally, the squark contribution becomes

$$
\begin{aligned}
\hat{\sigma}^{\tilde{q}} \equiv \hat{\sigma}_{H_{1}+H_{2}}^{\tilde{q}}+\hat{\sigma}_{H_{1}+H_{2}}^{q-\tilde{q}} \rightarrow & \frac{\alpha^{2} \alpha_{s}^{2}}{128 \pi \sin ^{4} \theta_{W}}\left(\frac{M_{\ell} M_{D}}{M_{W}^{2}}\right)^{2} \\
& \times \beta_{\ell}^{3} \frac{1}{\cos ^{4} \beta} \frac{1}{\hat{s}}\left(\frac{M_{D}^{2}}{\hat{s}}-\frac{1}{3}\right) .
\end{aligned}
$$

For small splittings of the quark masses in the doublet, the $Z^{0}$ contribution vanishes. We can see that the squark contribution goes like $1 / \hat{s}$ and is small for large values of the lepton-pair invariant mass. Therefore, the dominant contribution comes from the quark loop, involving Higgsboson exchange $\left(\hat{\sigma}^{q}\right)$. If we compare the prediction of the MSSM with the result of the standard model (SM) for the reaction $g g \rightarrow \ell^{+} \ell^{-}$[2], in the same limit, we can see that

$$
\frac{\hat{\sigma}_{\mathrm{MSSM}}}{\hat{\sigma}_{\mathrm{SM}}} \propto \frac{1}{\cos ^{4} \beta} .
$$

Therefore, large values of $\tan \beta$ lead to an important increase in the lepton production in the MSSM compared with the SM.

\section{RESULTS AND CONCLUSIONS}

The rapidity distribution for the process $p p \rightarrow g g \rightarrow$ $\ell^{+} \ell^{-}$is related to the subprocess $\left(g g \rightarrow \ell^{+} \ell^{-}\right)$total cross section $(\hat{\sigma})$ through

$$
\frac{d \sigma}{d y}=\int_{\tau_{\min }}^{e^{-2|y|}} d \tau G\left(\sqrt{\tau} e^{y}, Q^{2}\right) G\left(\sqrt{\tau} e^{-y}, Q^{2}\right) \hat{\sigma}(\tau s)
$$


where $\tau=\hat{s} / s\left(\tau_{\min }=4 M_{\ell}^{2} / s\right)$, with $s$ being the centerof-mass energy of the $p p$ system, and $G\left(x, Q^{2}\right)$ is the gluon structure function. Since this rapidity distribution is flat around $y=0$, its relation to the total cross section is immediate, and we shall initially analyze this quantity at zero rapidity.

Figure 2 presents the rapidity distribution at $y=0$ for the reaction $p p \rightarrow g g \rightarrow \ell^{+} \ell^{-}$, where we used the gluon distribution function given by Duke and Owens [11]. In order to understand the influence of the different parameters, we chose different values for the $H_{1}$ mass and $\tan \beta$, keeping fixed the quark and squark masses. Since the squark masses do not play a significant role in the process, in the numerical calculations we assumed that all the squarks are degenerated with $M_{\tilde{q}}=200 \mathrm{GeV}$. We can see that there are bumps due to $H_{1}$ and $H_{3}$ resonances for $M_{\ell}<100 \mathrm{GeV}$ and $M_{H_{1}}=200 \mathrm{GeV}$. These peaks occur when the width $\Gamma\left(H_{i} \rightarrow \ell^{+} \ell^{-}\right)$is maximum as a function of the lepton mass. They appear roughly at $M_{\ell}=M_{H_{i}} / \sqrt{n_{i}}$, where $n_{i}=10$ for $i=1,2$ and $n_{i}=6$ for $i=3$, and they end for $M_{H_{i}}<2 M_{\ell}$. Comparing the result with the Drell-Yan contribution for the lepton pair production $\left(p p \rightarrow q \bar{q} \rightarrow \ell^{+} \ell^{-}\right)$, we note that the gluonfusion mechanism is more important for $M_{\ell}>50 \mathrm{GeV}$, provided that $\tan \beta>2$.

We compare in Fig. 3 the predictions of the standard model and the MSSM for the heavy-lepton-pair production. We assumed different values for the masses of the fourth-generation quarks $\left(M_{U}, M_{D}\right)=(150,150) \mathrm{GeV}$ and $(150,650) \mathrm{GeV}$. The result for the MSSM is less sensitive to the mass splitting of the doublet than the standard model one, since in the former the contribution of the $Z^{0}$ is not significative. The result for the MSSM $(\tan \beta=5)$ is $\sim 50$ times larger than the standard model (SM) one for $M_{\ell} \simeq 100-200 \mathrm{GeV}$.

In order to present the enhancement of the cross section of the MSSM in relation to the SM result, we plotted

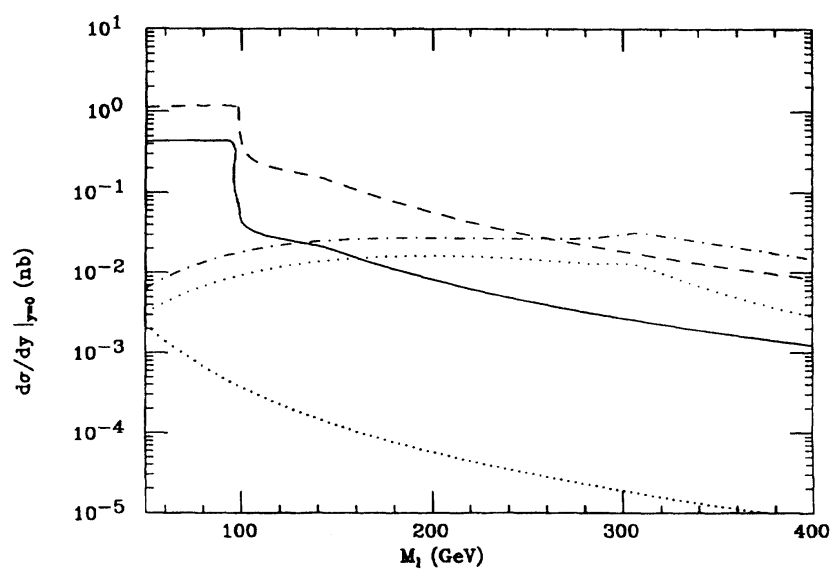

FIG. 2. Rapidity distribution at $y=0$ for the gluon fusion contribution to lepton production at the SSC in the MSSM as a function of the lepton mass. We assumed a fourth generation of quarks with mass $M_{U}=300 \mathrm{GeV}$ and $M_{D}=150 \mathrm{GeV}$. The different curves stand for $M_{H_{1}}=200 \mathrm{GeV}(\tan \beta=3$, solid line; $\tan \beta=5$, dashed line), and $M_{H_{1}}=600 \mathrm{GeV}$ $(\tan \beta=3$, dotted line; $\tan \beta=5$, dot-dashed line). We have also plotted the Drell-Yan contribution (lower dotted curve).

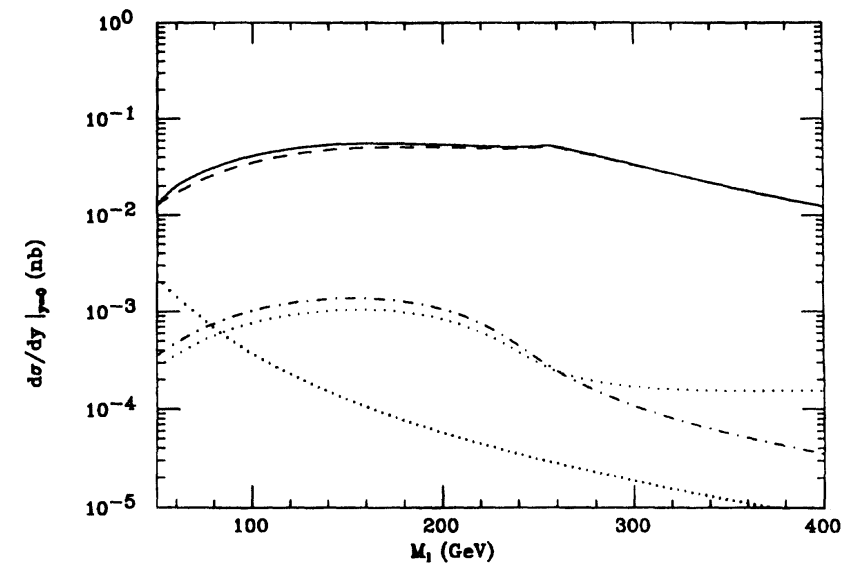

FIG. 3. Rapidity distribution at $y=0$ for the gluon-fusion contribution to lepton production at the SSC in the standard model (SM) and in the MSSM as a function of the lepton mass. We took for the Higgs-boson masses: $M_{H_{S M}}=M_{H_{1}}=$ $500 \mathrm{GeV}$ and $\tan \beta=5$. For the fourth generation of quarks we assumed $M_{D}=150 \mathrm{GeV}$ and $M_{U}=150 \mathrm{GeV}$ (MSSM, solid line; SM, dot-dashed line), and $M_{U}=650 \mathrm{GeV}$ (MSSM, dashed line; SM, dotted line). We have also plotted the DrellYan contribution (lower dotted curve).

in Fig. 4 the ratio

$$
R \equiv \frac{\left(d \sigma_{\mathrm{MSSM}} / d y\right)_{y=0}}{\left(d \sigma_{\mathrm{SM}} / d y\right)_{y=0}} \cos ^{4} \beta
$$

as a function of the lepton mass for several $\tan \beta$. We can observe that for large lepton masses this quantity is $\sim 0.5$, and is almost independent of $M_{\ell}$ and $\tan \beta$. This effect reflects the behavior of the elementary cross section discussed in the previous section. Moreover, this enhancement is also present in any two-Higgs-doublet models since this behavior does not depend on the presence of squarks in the loop.

In order to estimate the number of events we evaluate



FIG. 4. Ratio $R$ [Eq. (15)] as a function of the lepton mass at the SSC energies. We assumed $M_{U}=M_{D}=150 \mathrm{GeV}$ for the fourth generation of quarks and $M_{H_{\mathrm{SM}}}=M_{H_{1}}=100$ $\mathrm{GeV}$. The curves are for $\tan \beta=2$ (dotted line), $\tan \beta=5$ (solid line), and $\tan \beta=10$ (dashed line). 
the total cross section

$$
\sigma=\int_{\ln \sqrt{\tau_{\min }}}^{-\ln \sqrt{\tau_{\min }}} d y \frac{d \sigma}{d y}
$$

In Fig. 5 we present the result for the total cross section, for the proton-proton center-of-mass energies of $\sqrt{s}=16$ GeV [CERN Large Hadron Collider (LHC)] and $\sqrt{s}=$ $40 \mathrm{GeV}$ [Superconducting Super Collider (SSC)]. Taking into account the expected integrated luminosity for LHC $\left(10^{5} \mathrm{pb}^{-1} / \mathrm{yr}\right)$, and SSC $\left(10^{4} \mathrm{pb}^{-1} /\right.$ year $)$, we can expect a total of the order of $10^{5}$ heavy-lepton pairs produced per year in both machines.

The decay modes of charged heavy leptons depend on the mass difference $\Delta=M_{\ell}-M_{N_{\ell}}$, where $M_{N_{\ell}}$ is the heavy neutrino mass. If $\Delta>M_{W}$ the main decay mode of the charged heavy lepton is into a real $W$ and $N_{\ell}$, otherwise it exhibits a three-body decay, involving a virtual $W$. Requiring that the $W$ 's decay leptonically, the signal for a $\ell^{+} \ell^{-}$pair is $l^{+} l^{-}+/ p_{T}(l=\mu, e)$. The processes that have the same signature of this signal are $p p \rightarrow \tau^{+} \tau^{-}, W^{+} W^{-}$, and $Z^{0} Z^{0}$. The last final state can easily be distinguished by measuring the invariant mass of the charged-lepton pair.

The study of the signal and the background for the production of heavy charged leptons, within the standard model, was done in Refs. $[12,13]$. For $\Delta>M_{W}$, Hinchliffe [13] showed that it is possible to extract the leptonic signal by imposing a cut on the azimuthal angle $(\phi)$ that the two charged leptons make between themselves in the transverse plane. It was also shown that the only limitation for the discovery was the total number of events, i.e., the machine luminosity. Since, as we showed, the cross section is higher in the MSSM, the discovery of a heavy charged lepton can be made up to the masses that saturate the unitarity bounds, provided that $\Delta>M_{W}$.

On the other hand, for $\Delta<M_{W}$, it was shown in Ref. [12] that, in the case of the standard model production of heavy leptons, it is very hard to extract the signal from the background. For the MSSM production, we have a larger number of $\ell^{+} \ell^{-}$pairs being produced, indicating that it might be possible also to detect intermediate mass heavy leptons. However, this scenario can only be cleared by a careful Monte Carlo analysis of the signal and background.

To conclude we would like to remind that gluon-gluon fusion is also an important mechanism for the production of fourth-generation heavy neutrinos $(N)$. The cross section for the process $p p \rightarrow N \bar{N}$ was evaluated for the standard model in Ref. [14]. A similar analyses to the one of Sec. II shows that the ratio (13) for the process $g g \rightarrow N \bar{N}$ is now proportional to $1 / \sin ^{4} \beta$, being consequently enhanced for small values of $\tan \beta$. Nevertheless, $\tan \beta>1$ is preferred in model building [15], which means that there will be no enhancement for these processes in the framework of the MSSM.

\section{ACKNOWLEDGMENTS}

Two of us (O.J.P.E. and S.F.N.) are very grateful for the kind hospitality of the Institute for Elementary Parti- (a)

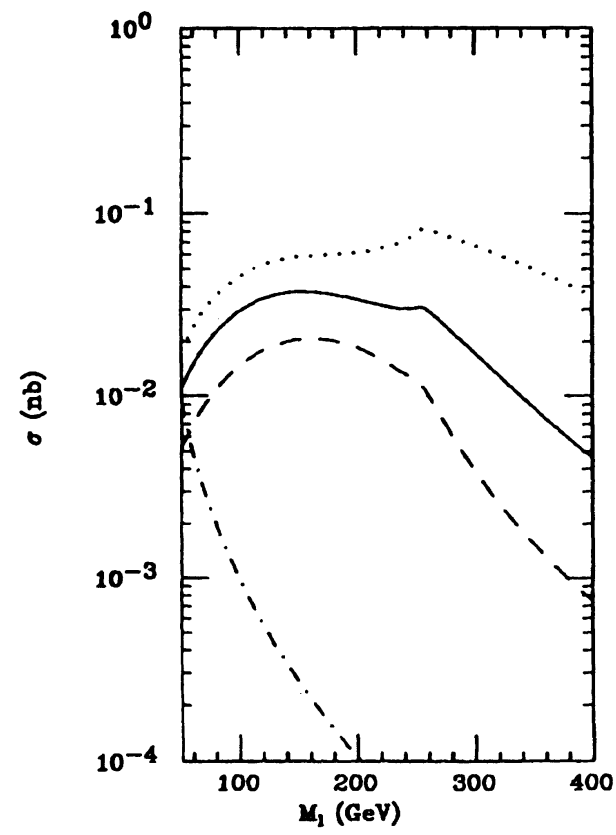

(b)

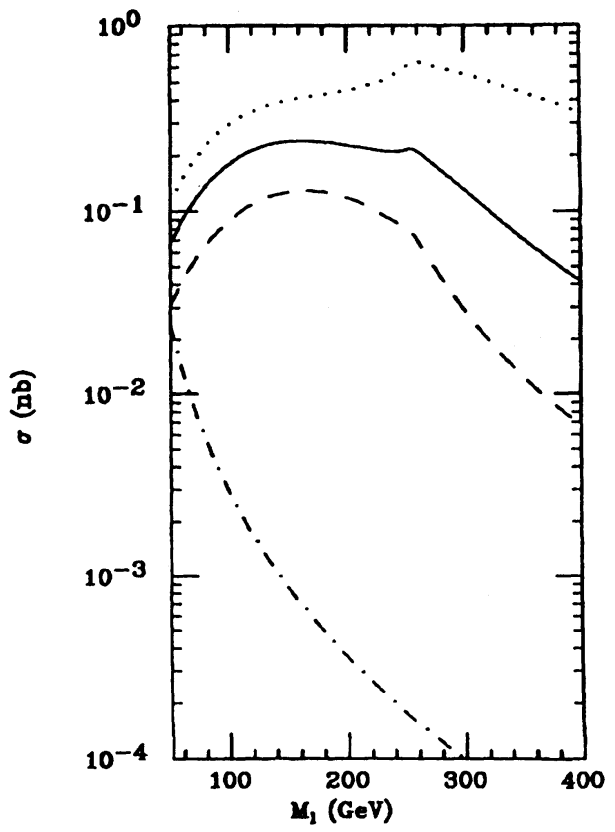

FIG. 5. Total cross section for the gluon-fusion contribution to lepton production in the MSSM as a function of the lepton mass. We considered the Higgs-boson mass: $M_{H_{1}}=500 \mathrm{GeV}$. For the fourth generation of quarks we assumed $M_{D}=150 \mathrm{GeV}$ and $M_{U}=300 \mathrm{GeV}$. The curves are for the center-of-mass energy of (a) $\sqrt{s}=16 \mathrm{TeV}$ (LHC) and (b) $\sqrt{s}=40 \mathrm{TeV}$ (SSC) $[\tan \beta=3$ (dashed line), $\tan \beta=5$ (solid line), and $\tan \beta=10$ (dotted line)]. We have also plotted the standard model result for the gluon fusion for $M_{H_{S M}}=500 \mathrm{GeV}$ (lower solid line) and the Drell-Yan contribution (lower dot-dashed curve). 
cle Physics Research, University of Wisconsin-Madison, where part of this work was done. This work was partially supported by Conselho Nacional de Desenvolvimento Científico e Tecnológico (CNPq) and Fundação de Amparo à Pesquisa do Estado de São Paulo (FAPESP).

\section{APPENDIX}

The loop integrals involved in the evaluation of the elementary cross section of Sec. II can be expressed in terms of the function $I_{i}\left(\lambda_{i}\right)$ which is defined through

$$
I_{i} \equiv I_{i}\left(\lambda_{i}\right)=\int_{0}^{1} \frac{d x}{x} \ln \left[1-\frac{(1-x) x}{\lambda_{i}}\right]=\left\{\begin{array}{l}
-2\left[\arcsin \left(\frac{1}{2 \sqrt{\lambda_{i}}}\right)\right]^{2}, \lambda_{i}>\frac{1}{4}, \\
\frac{1}{2} \ln ^{2}\left(\frac{r_{ \pm}}{r_{-}}\right)-\frac{\pi^{2}}{2}+i \pi \ln \left(\frac{r_{ \pm}}{r_{-}}\right), \quad \lambda_{i}<\frac{1}{4},
\end{array}\right.
$$

with $r_{ \pm}=1 \pm\left(1-4 \lambda_{i}\right)^{1 / 2}$ and $\lambda_{i}=m_{i}^{2} / \hat{s}$. Here, $i=q, \tilde{q}$ stands for the particle (quark or squark) running in the loop. Assuming that these particles are heavy, i.e., in the large- $\lambda_{i}$ limit, we have

$$
\lambda_{i} I_{i} \rightarrow-\frac{1}{2}, \quad \lambda_{i}\left[2+\left(4 \lambda_{i}-1\right) I_{i}\right] \rightarrow \frac{1}{3} .
$$

We wrote the couplings of the scalar Higgs bosons $\left(H_{1,2}\right)$ to the fermions $(f=u, d, \ell)$ as $i g m_{q} \eta_{f}^{(i)} / 2 M_{Z}$. The constants $\eta_{f}^{(i)}$ are functions of the mixing angles $\alpha$ and $\beta$ :

$$
\begin{aligned}
& \eta_{u}^{(1)}=\frac{\sin \alpha}{\sin \beta}, \quad \eta_{d}^{(1)}=\frac{\cos \alpha}{\cos \beta}=\eta_{\ell}^{(1)}, \\
& \eta_{u}^{(2)}=\frac{\cos \alpha}{\sin \beta}, \quad \eta_{d}^{(2)}=-\frac{\sin \alpha}{\cos \beta}=\eta_{\ell}^{(2)} .
\end{aligned}
$$

In the case of the pseudoscalar Higgs boson $\left(H_{3}\right)$, we wrote the vertex with the fermions as $g m_{q} \eta_{f}^{(i)} \gamma_{5} / 2 M_{Z}$, with

$$
\eta_{u}^{(3)}=\frac{\cos \beta}{\sin \beta}, \quad \eta_{d}^{(3)}=\frac{\sin \beta}{\cos \beta}=\eta_{l}^{(3)} .
$$

We also defined the squark-fermions couplings as

$\tilde{\eta}_{\tilde{u}_{L}}^{(1)}=\left(\frac{1}{2}-\frac{2}{3} \sin ^{2} \theta_{W}\right) \cos (\alpha+\beta)+\left(\frac{m_{u}}{M_{Z}}\right)^{2} \frac{\sin \alpha}{\sin \beta}$

$\tilde{\eta}_{\tilde{u}_{R}}^{(1)}=\frac{2}{3} \sin ^{2} \theta_{W} \cos (\alpha+\beta)+\left(\frac{m_{u}}{M_{Z}}\right)^{2} \frac{\sin \alpha}{\sin \beta}$,

$\tilde{\eta}_{\tilde{d}_{L}}^{(1)}=-\left(\frac{1}{2}-\frac{1}{3} \sin ^{2} \theta_{W}\right) \cos (\alpha+\beta)+\left(\frac{m_{d}}{M_{Z}}\right)^{2} \frac{\cos \alpha}{\cos \beta}$,

$\tilde{\eta}_{\tilde{d}_{R}}^{(1)}=-\frac{1}{3} \sin ^{2} \theta_{W} \cos (\alpha+\beta)+\left(\frac{m_{d}}{M_{Z}}\right)^{2} \frac{\cos \alpha}{\cos \beta}$,

$\tilde{\eta}_{\tilde{u}_{L}}^{(2)}=-\left(\frac{1}{2}-\frac{2}{3} \sin ^{2} \theta_{W}\right) \sin (\alpha+\beta)+\left(\frac{m_{u}}{M_{Z}}\right)^{2} \frac{\cos \alpha}{\sin \beta}$,

$\tilde{\eta}_{\tilde{u}_{R}}^{(2)}=-\frac{2}{3} \sin ^{2} \theta_{W} \sin (\alpha+\beta)+\left(\frac{m_{u}}{M_{Z}}\right)^{2} \frac{\cos \alpha}{\sin \beta}$,

$\tilde{\eta}_{\tilde{d}_{L}}^{(2)}=\left(\frac{1}{2}-\frac{1}{3} \sin ^{2} \theta_{W}\right) \sin (\alpha+\beta)-\left(\frac{m_{d}}{M_{Z}}\right)^{2} \frac{\sin \alpha}{\cos \beta}$,

$\tilde{\eta}_{\tilde{d}_{R}}^{(2)}=\frac{1}{3} \sin ^{2} \theta_{W} \sin (\alpha+\beta)-\left(\frac{m_{d}}{M_{Z}}\right)^{2} \frac{\sin \alpha}{\cos \beta}$.
[1] The LEP Collaborations: ALEPH, DELPHI, L3, and OPAL, Phys. Lett. B 276, 247 (1992).

[2] S. S. D. Willenbrock and D. A. Dicus, Phys. Lett. 156B, 429 (1985).

[3] H. E. Haber and G. L. Kane, Phys. Rep. 117, 75 (1985).

[4] J. Gunion and H. E. Haber, Nucl. Phys. B272, 1 (1986); B278, 449 (1986).

[5] ALEPH Collaboration, D. Decamp et al., Phys. Lett. B 265, 475 (1991).

[6] OPAL Collaboration, M. Akrawy et al., Phys. Lett. B 252, 290 (1990).

[7] We are grateful to D. Zeppenfeld for calling our attention to this point.
[8] D. A. Dicus and S. Nandi, Phys. Rev. D 31, 611 (1985); R. Casalbuoni et al., Phys. Lett. B 191, 409 (1987).

[9] M. S. Chanowitz, M. A. Furman, and I. Hinchliffe, Nucl. Phys. B153, 402 (1979).

[10] C. N. Yang, Phys. Rev. 77, 242 (1950).

[11] D. Duke and J. Owens, Phys. Rev. D 30, 49 (1984).

[12] V. Barger, T. Han, and J. Ohnemus, Phys. Rev. D 37, 1174 (1988).

[13] I. Hinchliffe, Int. J. Mod. Phys. A 4, 3867 (1989).

[14] D. A. Dicus and P. Roy, Phys. Rev. D 44, 1593 (1991).

[15] J. F. Gunion, H. E. Haber, G. Kane, and S. Dawson, The Higgs Hunter's Guide (Addison-Wesley, Reading, MA, 1990). 